\title{
Incremental Weighted Mining based on RFM Analysis for Recommending Prediction in u-Commerce
}

\author{
Young Sung Cho', Song Chul Moon ${ }^{2}$, In-Bae $\mathrm{Oh}^{3}$, \\ Jung-Hoon Shin ${ }^{4}$ and Keun Ho Ryu ${ }^{1}$ \\ ${ }^{1}$ Department of Computer Science, Chungbuk National University, \\ Cheongju, Korea \\ ${ }^{2}$ Department of Computer Science, Namseoul University, \\ Cheonan-city, Korea, Korea \\ ${ }^{3}$ Department of Industry Management, Chungbuk Health \& Science University, \\ Chungbuk, Korea \\ ${ }^{4}$ Department of Software Engineering, Chonbuk National University, \\ Jeonju, Korea \\ \{ youngscho@empal.com,moon@nsu.ac.kr,iboh@chsu.ac.kr,shinjh@jbnu.ac.kr, \\ khryu@dblab.chungbuk.ac.kr\}
}

\begin{abstract}
This paper proposes a new incremental weighted mining based on RFM((Recency, Frequency, Monetary) analysis for recommending prediction in u-commerce. Association rules search for the associated item set on large database. Association rules are frequently used by the marketing pattern analysis in e-commerce, recommendation to promote for selling a product in marketing. The proposing method can extract frequent items and create weighted association rules using incremental weighted mining based on RFM analysis rapidly when new data are added persistently in order to predict frequently changing trends by emphasizing the important items with high purchasability according to the threshold for creative weighted association rules in u-commerce. To verify improved better performance of proposing system than the previous systems, we carry out the experiments in the same dataset collected in a cosmetic internet shopping mall.
\end{abstract}

Keywords: Weighted Association Rules, RFM analysis, Mining using FP-tree, Incremental Mining

\section{Introduction}

Along with the advent of ubiquitous computing and the spread of intelligent portable device such as smart phone, PDA and smart pad has been amplified, a variety of services and the amount of information has also increased. It is becoming a part of our common life style that the demands for enjoying the wireless internet are increasing anytime or anyplace without any restriction of time and place [2, 4]. It is necessary for us to search the associated item set on large databases in order to recommend the proper item to be reflected by the marketing pattern analysis in e-commerce, among large item sets. The possession of intelligent recommendation system is becoming the company's business strategy. A recommendation system using data mining technique based on RFM to meet the needs of customers has been actually processed the research[1-5]. A existing recommendation system using association rules has the problem, such as delay of processing speed from a cause of 
frequent scanning a large data, scalability and accuracy as well. It is crucial to have different weights for different transactions in order to reflect their different importance and to adjust the mining results by emphasizing the important transactions. We can improve the performance of recommendation through incremental weighted mining based on RFM analysis for recommending prediction in u-commerce so as to be able to generate the association rules. As a result, we propose a new incremental weighted mining based on RFM analysis for recommending prediction in u-commerce under ubiquitous computing environment. The next section briefly reviews the literature related to studies. The section 3 is described a new method for personalized recommendation system in detail, such as system architecture with sub modules, the procedure of processing the recommendation, the algorithm for proposing system. The section 4 describes the evaluation of this system in order to prove the criteria of logicality and efficiency through the implementation and the experiment. In section 5, finally it is described the conclusion of paper and further research direction.

\section{Relative Works}

\subsection{RFM Analysis}

Segmentation by product usage uses a method called purchase RFM analysis to segment customers on the basis of how long since they made purchases, how frequently they make purchases, and how much money they spend. It is generally known in database marketing and direct marketing. It is easy for us to recommend the item with high purchasability using the customer's score and the item's score according to the level of customer. The RFM score can be a basis factor how to determine purchasing behavior on the internet shopping mall, is helpful to buy the item which they really want by the personalized recommendation. The RFM score will be shown how to determine the customer as follows, will be used in Table 3 . The variables $(\mathrm{A}, \mathrm{B}, \mathrm{C})$ are weights. The categories $(\mathrm{R}, \mathrm{F}, \mathrm{M})$ have five bins.

$$
\begin{array}{|l|l|}
\hline \mathrm{RFM}=\mathrm{A} \times \mathrm{R}+\mathrm{B} \times \mathrm{F}+\mathrm{C} \times \mathrm{M} & (1) \\
\hline
\end{array}
$$

The RFM score is correlated to the interest of e-commerce [2-5]. It is necessary for us to keep the analysis of RFM to be able to reflect the attributes of the item in order to find the items with high purchasability. In this paper, we can use weighted mining association rules with the weight based on the quantity of purchased data aggregated from the whole data with the item RFM score to recommend the item they really want exactly.

\subsection{Weighted Association Rules}

Association rules mining is one of the most important and widespread data mining techniques. It was first introduced by Agrawal[6]. Association rule mining is a common technique for performing market basket analysis. Association rule(AR) is the discovery of sets of products usually purchased together by many independent buyers. To select interesting rules from the set of all possible rules, the best-known constraints on various measures of significance and interest can be used. An association rule $X \rightarrow Y$ represents a relationship between the sets of items $\mathrm{X}$ and $\mathrm{Y}$. Each item $\mathrm{I}$ is an atom representing a particular object. The relation is characterized by two measures: support and confidence of the rule. The constraints are minimum thresholds on support and confidence. The traditional association rule mining employs the support measure, which treats every transaction equally. However, in our real world data sets, the weight importance of a pattern may vary frequently due to some 
unavoidable situations. Usually in an association rules, it is expressed in the form of the rule $X \rightarrow Y$. The rule of $X \rightarrow Y$ means that the transaction including the item of $X$ tends to include the itemsets. And then in an weighted association rules, the w-support of an weighted association rule $X \rightarrow Y$ is defined as

$$
\operatorname{WSUPP}(\mathrm{X} \rightarrow \mathrm{Y})=\operatorname{WSUPP}(X \cup Y)
$$

and the w-confidence is

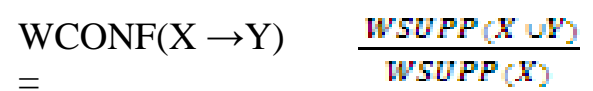

Basically, w-support measures how significantly $\mathrm{X}$ and $\mathrm{Y}$ appear together; w-confidence measures how strong the rule is. An weighted association rule mining becomes an important research issue in data mining and knowledge discovery by considering different weights for different items. It is necessary to consider these dynamic changes in different application area such as retail market basket data analysis. Much effort has been dedicated to association rule mining with pre-assigned weights [8,9]. It is crucial to have different weights for different transactions in order to reflect their different importance and adjust the mining results by emphasizing the important transactions.

\subsection{Mining using FP-tree}

The FP-tree is a compact representation of all relevant frequency information in a database. To construct the FP-tree, the first scan is to find all frequent items by an initial scan of the database. Then, these items are inserted into the header table in decreasing order of their count. In the second scan, as each transaction is scanned, the set of frequent items in it are inserted into the FP-tree as a branch. Every branch of the FP-tree represents a frequent itemset and the nodes along the branches are stored in decreasing order of frequency of the corresponding items with leaves representing the least frequent items. Compression is achieved by building the tree in such a way that overlapping itemsets share prefixes of the corresponding branches. An FP-tree T has a header table, T:header, associated with it. Single items and their counts are stored in the header table in decreasing order of their frequency. The entry for an item also contains the head of a list that links all the corresponding nodes of the FP-tree. Compared with breadth-first algorithms such as Apriori and its variants, which may need as many database scans as the length of the longest pattern, the FP-growth method only needs two database scans when mining all frequent itemsets. Now, the constructed FPtree contains all frequency information of the database. Mining the database becomes mining the FP-tree. The algorithm for construction of FP-tree mining [2] as following Table 1. The procedure of insertion for compact pattern tree in FP-tree mining [2] as following Table 2.

Table 1. The Algorithm for Construction of FP-tree Mining

\begin{tabular}{l}
\hline Input : (1) Expression data set D ; (2) minimum support Min $n_{\text {sup }}$ \\
Output : FP-tree corresponding to and satisfying Min sup \\
\hline 1) Scan D once and collect the set of frequent items F and their supports. \\
2) Sort F in support descending order as L, the list of frequent items. \\
3) If several items have the same support, and their names are numbers, sort the items in \\
ascending order of their names. \\
4) Create the root R of a new FP-tree and label it as "null".
\end{tabular}


5) Create frequent-item header table with $|F|$ entries. Set all head of node-link pointers to null.

6) for each transaction data $d \in D$ do // Read $D$ the second time.

7) Select only frequent items of $d$ into a record $P$;

8) Sort $P$ in the order of $L$;

9) Call insert_tree $\left(P, c_{d}, R\right)$;

10) end for

Table 2. The Procedure of Insertion for Compact Pattern Tree

Procedure insert_tree $(P, c, R)$;

1) Let $P=[p \mid P-p]$, where $p$ is the first element of $P$, and $P-p$ is the remaining list.

2) if $R$ has a child $N$ such that $N$.item_name $=p$ then

3) N.count $=$ N.count +1 ;

4) else \{

5) create a new node $N$;

6) $N$.count $=1$; N.item_name $=p$;

8) $N$.parent $=R$; N.node-link $=H(p)$.head;

9) $H(p)$.head = $N$;

10) $\}$

11) $H(p)$.count $=H(p)$.count +1 ;

12) if $P-p \neq \varphi$ then

13) Call insert_tree $(P-p, c, N)$ recursively.

\subsection{Incremental Mining}

In data mining, association rules play an important role and have been applicable in many areas. There are two problems in mining association rules. One is finding frequent itemsets. The other is using those itemsets to generate the rules. After the frequent itemsets have been recognized, the corresponding rules may be derived easily. The knowledge discovered from transactional databases in e-commerce has to be maintained, and an incremental updating technique needs to be developed for maintaining the discovered association rules from these databases. The concept of association rules has been introduced to solve the problem of handling time series by including time expressions into association rules. D.W Cheung introduced a new algorithm called the Fast Update algorithm(FUP)[7], for efficient maintenance of discovered association rules when new transactions are added. It's similar in its frame work to that of Apriori [6]. It contains a number of iterations starting at size-one itemsets; at each iteration, all the large itemsets of the same size are founded. The candidate sets at each iteration are generated based on the large itemsets founded in the previous iteration. FUP introduces to handle the incremental database and to update the discovered rules. At each iteration the support of size-k frequent itemsets are updated against the incremental database to filter out the loser. Only the incremental database has to be scanned to do this filtering. While scanning the incremental database, a set of candidates is extracted along with their support from the incremental database. 


\section{Our Proposal for Recommending Prediction in u-commerce}

\subsection{System Architecture}

We can depict the system configuration concerning the recommendation system using incremental weighted mining to predict item with high purchasability under ubiquitous computing environment which is required by real time accessibility and agility. This system had four agent modules which have the analytical agent, the recommendation agent, the learning agent, the data mining agent in the internet shopping mall environment. We observed the web standard in the web development, so developed the interface of internet to use full browsing in mobile device. As a matter of course, we can use web browser in wired internet to use our recommendation system. We can use the system under WAP in mobile web environment by using feature phone as well as using the internet browser such as safari browser of iPhone and Google chrome browser based on android so as to use our system by using smart phone.

\subsection{Incremental Weighted Mining based on RFM Analysis}

In this part, there are two issues about our proposal for recommendation system in ucommerce. It is necessary for us to predict a good recommendation in order to promote a recommendation for selling a product in marketing. One is a new weighted mining algorithm using FP-tree, been different from the papers. We can use a new weighted method of the quantity of purchased data aggregated from the whole data with the item RFM score to join the customer information for pre-processing so as to be possible to recommend the item with purchasability. Much effort has been dedicated to association rule mining with pre-assigned weights $[8,9]$. However, existing weighted methods, though, are not able to estimate the type of importance and adjust the mining results by emphasizing the important transactions. Our algorithm for weighted mining based on RFM analysis can consider the situation where the weight / importance of a pattern may vary dynamically in u-commerce on the real world. It is necessary for us to consider the quantity of purchased data by each rank of RFM score in order to have different weights for different transactions and to generate weighted association rules through weighted mining based on RFM analysis to predict items for recommending in u-commerce according to the threshold for creative weighted association rules with w-support, w-confidence and w-lift. As a matter of course, our weighted method is quite different from existing weighted methods. The procedural algorithm for weighted mining based on RFM analysis for recommending prediction in u-commerce is depicted as the following Table 3.

Table 3. The Procedural Algorithm for Weighted Mining based on RFM Analysis

Step $1:$ The RFM score of item is computed so as to reflect the attributes of the item, consists of three attributes, each attribute has five bins divided by each 20\%, exact quintile. As a result, we can make the RFM scores of items.

Step 2 : We can aggregate the quantity of purchased data by each interval RFM scores divided by each $20 \%$, which is aggregated counts of distribution from the whole data with the item RFM score, make the rate of weight.

Step 3 : We can make the rate of weight for items through aggregated counts of distribution. This is the value of weight based on the quantity of purchased data.

Step 4 : Association rules are created by WMAR using FP-tree

Step 5 : Wsupport $=\sum_{\mathbb{1}}^{W}$ Weight $/ N \mathrm{X}$ Support count $\quad / * N$ is numbers of item in the rules */ 
Step 6 : We can create creative association rules with support, confidence and lift through weighted mining with the weight based on the quantity of purchased data

Step 7 : We can scan whole database(sale) and generate association rules through weighted mining with the weight based on the quantity of purchased data.

Step 8: We can recommend the item with high purchasability according to the threshold for creative association rules with support, confidence and lift.

The other is an incremental mining rapidly when new data are added persistently in emerging data under ubiquitous computing environment. The association rules mining has the problem, which is suffered from inefficiency by reprocessing of the data which have already been processed in which new data are added persistently [5]. It is necessary for us to overcome non real time limitation of association rule discovery requiring very high overhead. Existing recommendation system is not able to reflect exact attributes of item. We can use the method of data processing for mining, which is new data added persistently, We can gather lots of data for two weeks to insert into the knowledge database of association rules after an interval of two weeks. In this paper we introduce a novel algorithm (IWMAR: Incremental Weighted Mining Association Rules) using FP-tree. You had better to refer the Table 4 of notations description to understand the procedural algorithm for weighted mining based on RFM analysis as the following Table 5. The proposed can extract frequent items and create association rules using incremental mining rapidly when new data are added persistently in emerging data environment. We can describe our proposal algorithm(IWMAR: Incremental Weighted Mining Association Rules) based on RFM analysis, a successful structure to mine frequent patterns. As a matter of course, we can use mining association rules with the weight based on the quantity of purchased data aggregated from the whole data(sale) with the item RFM score. The procedural algorithm for IWMAR is depicted as the following Table 5.

Table 4. Notations Description

\begin{tabular}{|l|l|}
\hline \multicolumn{1}{|c|}{ Notation } & \multicolumn{1}{c|}{ Description } \\
\hline \hline $\mathrm{DB}$ & Original database \\
\hline $\mathrm{db}$ & Incremental database \\
\hline $\mathrm{UD}$ & Updated database \\
\hline $\mathrm{D}$ & Number of Transaction in db \\
\hline $\mathrm{S}$ & minimun support in db \\
\hline Lk & Frequent k-itemsets in DB U db \\
\hline X.Supportdb & $\begin{array}{l}\text { Support count of item X in the } \\
\text { incremental database }\end{array}$ \\
\hline T-treedB & FP-tree of the original database \\
\hline T-treedb & $\begin{array}{l}\text { FP-tree of the incremental } \\
\text { database }\end{array}$ \\
\hline T-treeud & FP-tree of the updated database \\
\hline
\end{tabular}

\section{Table 5. Procedural ALgorithm for Processing} by IWMAR

Input : sale, old_db $\left(T-t_{r e e_{D B}}\right)$, new_db $\left(T-t_{\text {tree }}\right.$ db $)$,

Minimum Support threshold. // sale is whole data

Output : now_db $\left(T-t_{r} \in e_{\mathrm{WD}}\right)$, AR_tbl;

Begin

if (not exist (now_db $\left(T-\right.$ tree $\left.\left.e_{\mathrm{WD}}\right)\right)$ )

now_db $\left(T-\right.$ tree $\left._{\text {oD }}\right) \leftarrow$ WMAR(sale);

// Weighted Mining Association Rule with Minimum Support

threshold using WMAR based on sale

Save (AR_tbl) $\leftarrow$ now_db $\left(T-\right.$ tree $\left.e_{\text {UD }}\right)$;

// create the Association Rules table

end if;

if (not exist (new_db $\left(T-\right.$ tree $\left.\left._{\mathrm{db}}\right)\right)$ ) exit;

Rename now_db $\left(T-t_{r e e_{\mathrm{DD}}}\right)$ to old_db $\left(T-\right.$ tree $\left._{D B}\right)$;

while (!= EOF(new_db()))

$\left\{\right.$ now_db $\left(T-\right.$ tree $\left.e_{\text {OD }}\right) \leftarrow$

update (old_db $\left(T-\right.$ tree $\left._{\mathrm{DB}}\right) \mathrm{U}$ new_db $\left(T-\mathrm{tree}_{\mathrm{OD}}\right)$ );

// create now_db( ) using WMAR

Save $\left(A R \_t b l\right) \leftarrow$ now_db $\left(T-\right.$ tree $\left._{\mathrm{OD}}\right)$;

//create the Association Rules table for recommendation newly \}

End: 


\subsection{The Procedural Algorithm for Recommendation}

The system can search the information in the cluster selected by using the code of classification in users' information. It can scan the preference as the average of brand item in the cluster, suggest the brand item in item category selected by the highest probability for preference as the average of brand item. This system can create the list of recommendation with TOP-N of the highest preference of item to recommend the item with purchasability efficiently. This system can recommend the items with efficiency, are used to generate the recommendable item according to the basic threshold for weighted association rules, with support, confidence and lift. It can recommend the associated item to TOP-N of recommending list if users want to have the cross-selling or up-selling. This system takes the cross comparison with purchased data in order to avoid the duplicated recommendation which it has ever taken.

\subsection{The Analysis of Application for Weighted Mining for Recommendation}

In this section, we can have the experimental analysis of validity to do the process of mining for experimental evaluation. We have made two experimental tasks of mining process in order in the same condition to compare the result of mining association rules. One is the test of Ordinary mining association rules in original data(sale). The other is the test of proposing weighted mining association rules with the weight based on the quantity of purchased data aggregated from the whole data with the item RFM score so as to make the solution of mining improved by the performance as the metrics with support count, rule count, support, confidence and lift. The result of experimental test is the metrics of association rules with support, confidence and lift as the following Table 6. The result of proposing mining is higher average rates of association rules with support count, rule count, support, and lift than the ordinary mining even if the proposal is lower 6.892 in average of confidence than the original.

Table 6. The Result on the Performance for Mining

\begin{tabular}{|c|c|c|c|c|c|c|}
\hline & $\begin{array}{c}\text { sale } \\
\text { count }\end{array}$ & $\begin{array}{c}\text { support } \\
\text { count }\end{array}$ & $\begin{array}{c}\text { Rule } \\
\text { count }\end{array}$ & $\begin{array}{c}\text { average } \\
\text { sup_rate }\end{array}$ & $\begin{array}{c}\text { average } \\
\text { conff_rate }\end{array}$ & $\begin{array}{c}\text { average } \\
\text { lift_rate }\end{array}$ \\
\hline Proposal & 1,600 & 16,908 & 1,606 & 0.249 & 34.487 & 6.024 \\
\hline Ordinary & 1,600 & 15,570 & 406 & 0.227 & 41.379 & 5.233 \\
\hline
\end{tabular}

We use the same original data(sale), so both of method is similar processing time for mining at the first phase. And also, the result of proposing mining for processing time is faster than existing mining(Ordinary) because proposing system(Proposal) create association rules in Table 6 mining rapidly when new data are added persistently at the second phase. It is important for us to process the large databases in u-commerce on the real world. As a result of that, we can obtain the result of the performance of mining as the metrics with support count, rule count, support and lift though confident rate is less than the mining of original method. We can describe the evaluation of this system in order to prove the criteria of logicality and efficiency through the implementation and the experiment in next session 4. 


\section{The Environment of Implementation and Experiment \& Evaluation}

\subsection{Experimental Environment}

This system proposes an new incremental weighted mining based on RFM analysis for recommending Prediction in u-commerce under ubiquitous computing environment. In order to do that, we make the implementation for prototyping of the internet shopping mall which handles the cosmetics professionally and do the experiment. It is the environment of implementation and experiment below.

- OS: Windows XP SP2,

- Web Server: Apache 2.2.14 / WAP 2.0

- Server-Side Script : JSP/PHP 5.2.12

- XML/WML2.0/ HTML5.0/CSS3/JAVASCRIPT

- Database: MySQL 5.1.39

- J2SDK(1.7.0_11)

- MySQL JDBC

- J2SDK(1.7.0_11)

- jakarta-tomcat (5.0.28)

We have carried out the implementation and the experiment for proposing system through system design, we have finished the system implementation about prototyping recommendation system. It could be improved and evaluated to new system through the result of experiment with the metrics used in the field of information retrieval commonly [10], such as precision, recall, F-measure as comparing proposing system with existing method and system.

\subsection{Experimental Data for Evaluation}

We used 319 users who have had the experience to buy items in e-shopping mall, 580 cosmetic items used in current industry, 1600 results of purchased data recommended in order to evaluate the proposing system [4]. In order to do that, we make the implementation for prototyping of the internet shopping mall which handles the cosmetics professionally and do the experiment. We have finished the system implementation about prototyping recommendation system. We'd try to carry out the experiments in the same condition with dataset collected in a cosmetic internet shopping mall. It could be proved by the experiment through the experiment with learning data set for 12 months, testing data set for 3 months in a cosmetic cyber shopping mall [4]. The $1^{\text {st }}$ system of weighted mining using FP-tree, is proposing system called by "proposal”, the $2^{\text {nd }}$ system is the original system(O_ARM) using the ordinary association rules mining, the third system is existing system.

\subsection{Experiment \& Evaluation}

We can make the task of clustering of item category based on purchased data for preprocessing under ubiquitous computing environment. The proposing system's overall performance evaluation is precision, recall and F-measure for proposing system comparing than the original system which is proposed by ex-paper [1]. The performance was performed to prove the validity of recommendation and the system's overall performance evaluation. 
Table 7. The Result for Table of Precision, Recall, F-measure for Recommendation Ratio by each Cluster

\begin{tabular}{|l|l|l|l|l|l|l|l|l|l|}
\hline \multirow{2}{*}{ Cluster } & \multicolumn{3}{|l|}{ Proposal(W_ARM) } & \multicolumn{2}{l|}{ Original((O_ARM) } & \multicolumn{2}{l|}{ Existing } \\
\cline { 2 - 11 } & $\begin{array}{l}\text { Preci } \\
\text { sion1 }\end{array}$ & Recall1 & $\begin{array}{l}\text { F- } \\
\text { mea } \\
\text { sure1 }\end{array}$ & $\begin{array}{l}\text { Preci } \\
\text { sion2 }\end{array}$ & Recall2 & $\begin{array}{l}\text { F- } \\
\text { mea } \\
\text { sure2 }\end{array}$ & $\begin{array}{l}\text { Preci } \\
\text { sion3 }\end{array}$ & Recall3 & $\begin{array}{l}\text { F-me } \\
\text { asure3 }\end{array}$ \\
\hline \hline C1 & 35.24 & 70.00 & 46.88 & 46.20 & 55.70 & 45.90 & 48.79 & 31.32 & 35.64 \\
\hline C2 & 33.18 & 62.42 & 43.33 & 43.20 & 52.53 & 45.76 & 49.36 & 29.54 & 35.06 \\
\hline C3 & 34.00 & 65.66 & 44.80 & 40.99 & 23.93 & 30.05 & 44.26 & 21.81 & 27.65 \\
\hline C4 & 36.39 & 68.84 & 47.61 & 33.56 & 37.23 & 34.68 & 50.93 & 36.60 & 39.64 \\
\hline C5 & 45.78 & 66.60 & 54.26 & 53.94 & 27.27 & 34.90 & 47.41 & 26.81 & 32.26 \\
\hline C6 & 39.78 & 71.90 & 51.22 & 45.07 & 37.23 & 38.29 & 43.60 & 36.60 & 37.82 \\
\hline C7 & 42.52 & 75.49 & 54.40 & 64.08 & 28.45 & 37.19 & 46.68 & 25.19 & 30.28 \\
\hline C8 & 31.89 & 74.75 & 44.70 & 60.00 & 20.69 & 30.77 & 46.53 & 18.32 & 25.10 \\
\hline C9 & 44.90 & 80.23 & 57.57 & 73.85 & 62.50 & 64.42 & 67.23 & 55.34 & 57.10 \\
\hline
\end{tabular}
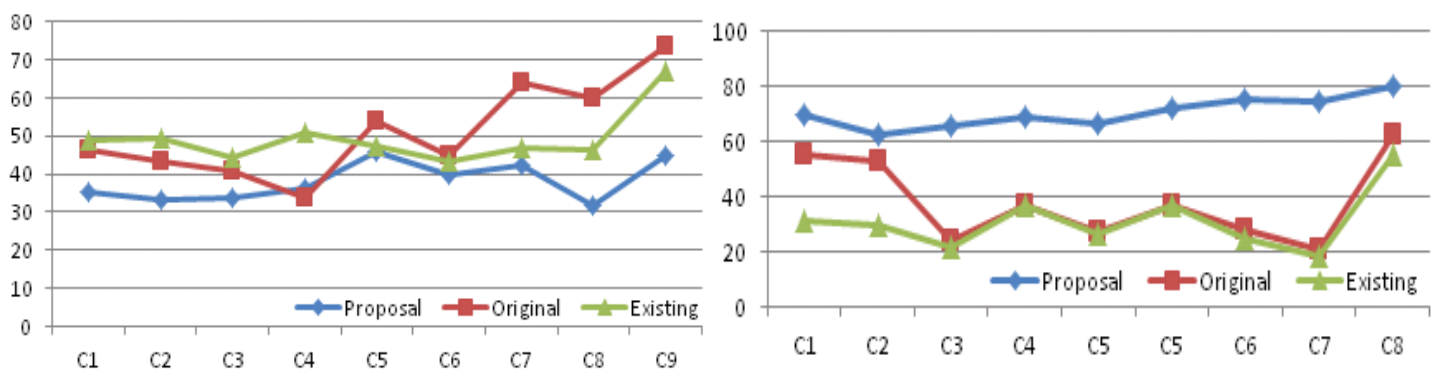

Figure 1. The Result of Recommending Figure 2. The Result of Recommending Ratio by Precision Ratio by recall

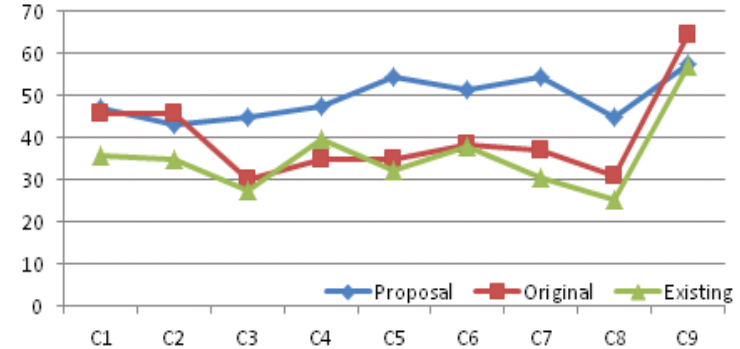

Figure 3. The Result of Recommending Ratio by F-measure

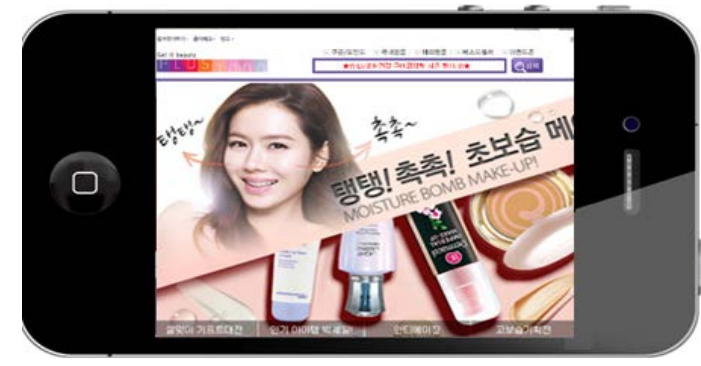

Figure 4. The Result of Recommending Items of Cosmetics

Above Table 7 presents the result of evaluation metrics(precision, recall and F-measure) for recommendation system. The weighted mining is improved better performance of proposing system than the original system proposed in the ex-paper[1]. The proposed higher $32.26 \%$ in recall even if it is lower $13.02 \%$ in precision than the original system, higher $9.2 \%$ in F-measure than the original system. As a result, we could have the recommendation system 
to be able to recommend the items with high purchasability. Above figure 4 is shown in the result of screen on a smart phone. The performance of proposing system was improved more counts of support and rule than the original system, it was especially worthy of notice, in the rule counts, had an effect about 4 times what the original system did before. As a result, it was efficient for us to recommend the items of association because it is strong cohesion of the attribute of item based association rules [11] with the weight based on the quantity of purchased data aggregated from the whole data with the item RFM score.

\section{Conclusion}

Recently u-commerce as a application field under fixed mobile convergence service environment required by real time accessibility and agility, is in the limelight [4]. A existing recommendation system using association rules has the problem, which is suffered from inefficiency by reprocessing of the data which have already been processed in incremental data in which new data are added persistently. We proposed a new incremental weighted mining for recommending Prediction in emerging data environment in order to improve the accuracy of recommendation with high purchasability. We have described that the performance of the proposing system with mining is improved better than the original system and existing system. To verify improved better performance of proposing, we carried out the experiments in the same dataset collected in a cosmetic internet shopping mall. It is meaningful to present a new methodology of weighted mining for recommendation system in emerging data under ubiquitous computing environment. Furthermore, it will be an interesting research area on the aspect of effectiveness for recommendation system if we apply weighted mining after SOM clustering using RFM analysis. The following research will be looking for recommending Prediction by SOM clustering approach to increase the efficiency and scalability.

\section{Acknowledgements}

This work $^{1)}$ was supported by the National Research Foundation of Korea(NRF) grant funded by the Korea government(MEST) (No. 2012-0000478) and this paper') was supported by funding of Namseoul University.

\section{References}

[1] Y. S. Cho, S. P. Jeong and K. H. Ryu, "Implementation of Personalized u-commerce Recommendation System using Preference of Item Category based on RFM”, the 6th International Conference on Ubiquitous Information Technologies \& Applications”, 6th International Conference on Ubiquitous Information Technologies \& Applications, (2011) December, pp. 109-114.

[2] Y. S. Cho and K. H. Ryu, "Personalized Recommendation System Mining based on RFM", 17th KSCI, vol. 2, (2012) February.

[3] Y. S. Cho, S. C. Moon, S. C. Noh and K. H. Ryu, "Implementation of Personalized recommendation System using k-means Clustering of Item Category based on RFM”, 2012 IEEE International Conference on Management of Innovation \& Technology Publication, (2012) June.

[4] Y. S. Cho, S. C. Moon and K. H. Ryu, "Mining Association Rules using RFM Scoring Method for Personalized u-Commerce Recommendation System in emerging data”, International Conferences, SecTech, CA, CES3 2012, Held in Conjunction with GST 2012, Communications in Computer and Information Science, Springer, (2012), pp. 190-198.

[5] Y. S. Cho, S. C. Moon and K. H. Ryu, "RFM based Incremental Frequent Patterns mining Method for Recommendation in e-Commerce”, KSCI, The Korea Society of Computer and Information Summer Conference 2012, (2012) July, pp 135-137.

[6] R. Agrawal, T. Imielinski and A. Swami, "Mining Association Rules between Sets of Items in Large Datasets”, Proc. ACM SIGMOD International Conference on Management of Data, (1995), pp. 207-216. 
[7] D. W. Cheung, J. Han, V. Neg and Y. Wong, "Maintenance of Discovered Association Rules in Large Databases: An Incremental Updating Technique”, The International Conference on Data Engineering, (1996), pp. 106-114.

[8] G. D. Ramkumar, S. Ranka and S. Tsur, "Weighted Association Rules: Model and Algorithm”, Proc. ACM SIGKDD, (1998).

[9] F. Tao, F. Murtagh and M. Farid, "Weighted Association Rule Mining Using Weighted Support and Significance Framework”, Proc. ACM SIGKDD 2003, (2003), pp. 661-666.

[10] J. L. Herlocker, J. A. Kosran, A. Borchers and J. Riedl, "An Algorithm Framework for Performing Collaborative Filtering, "Proceedings of the 1999 Conference on Research and Development in Information Research and Development in Information Retrival, (1999).

[11] M.-S. Shin, “An Alert Data Mining Framework for Intrusion Detection System”, Journal of Korea Academia-Industrial cooperation Society, vol. 12, no. 1, (2011).

\section{Authors}

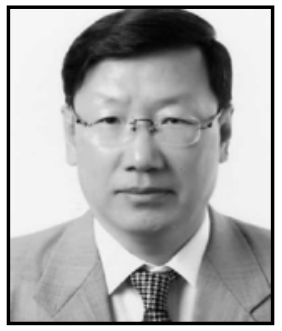

Young Sung Cho is a Researcher, Chief of R\&D Lab. in Comtree Co in Korea. His research interests include Time/Space DB, Machine Learning, Data mining, e-commerce, Web Service, Ubiquitous computing and DB Security. He has a MS in Computer Engineering from Yonsei University and a $\mathrm{PhD}$ in Computer Science from Chungbuk National University, email: youngscho@empal.com.

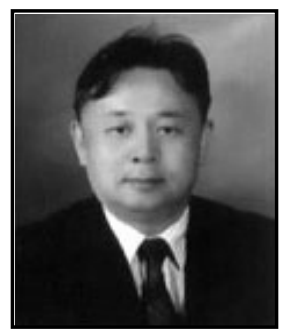

Song Chul Moon is a professor of Dept. of Computer Science at Namseoul University in Korea. His research interests include Information Management, Information Security, e-commerce, and Ubiquitous computing. He has a MS in Management Information Engineering from the Korea. Advanced Institute of Science and Technology and a PhD in Information Management from Kukmin University, email: moon@nsu.ac.kr.

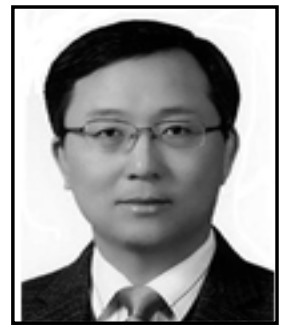

In-Bae Oh is a professor of Dept. of Industry Management at Chungbuk Health \& Science University in Korea. His research interests include Time/Space DB, GIS, Mobile DB, Virtual Reality. He has a BS in Computer Engineering from Hannam University, MS from Konkuk University and PhD from Chungbuk National University in Korea, email: iboh@chsu.ac.kr.

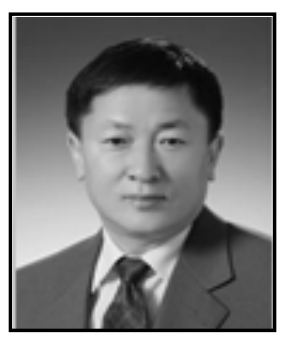

Jung Hoon Shin is a professor of Dept. of Software Engineering at Junbuk National University in Korea. His research interests include Operating System, Software Engineering, Data mining, Knowledge DB, and Machine Learning. He has a MS and PhD in Computer Science in Chungbuk National University, email: shinjh@jbnu.ac.kr. 


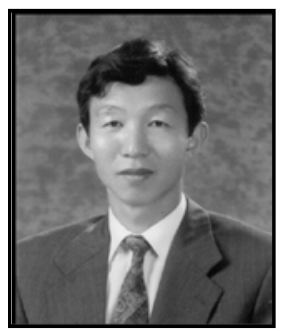

Keun Ho Ryu is a professor of Dept. of Computer Science at Chungbuk National University in Korea. His research interests include Time/Space DB, Temporal GIS, Knowledge based Information Retrieval, Data mining, Ubiquitous computing, Stream Data Processing, DB Security, Bio-Infomatics. He has a BS in Computer Engineering from SungSil University, $\mathrm{MS}$ and $\mathrm{PhD}$ from Yonsei University, email: khryu@dblab.chungbuk.ac.kr. 